\title{
无溶剂条件下方便、有效的方法合成 3-氰基-2-吡啶酮衍生物
}

\author{
荣良策*,a,b 刘丽华 ${ }^{a}$ 殷 姗 ${ }^{b}$ 夏 盛 ${ }^{b}$ 魏贤勇*,a 宗志敏 ${ }^{a}$ \\ $\left({ }^{a}\right.$ 中国矿业大学化工学院 徐州 221008) \\ ( ${ }^{b}$ 徐州师范大学化学化工学院 徐州 221116)
}

\begin{abstract}
摘要 芳醛、丙酮和氭乙酰胺为原料, 氢氧化钠为催化剂, 在无溶剂条件下加热反应有效地合成 2-吡啶酮衍生物, 本方 法具有反应时间短、条件温和、处理简单和环境友好等优点.
\end{abstract}

关键词 2-吡啶酮; 杂环; 无溶剂反应; 合成

\section{A Facile and Efficient Synthesis of 3-Carbonitrile Pyridin-2(1H)-one Derivatives under Solvent-Free Conditions}

\author{
Rong, Liangce ${ }^{*, a, b} \quad$ Liu, Lihua $^{a} \quad$ Yin, Shan $^{b} \quad$ Xia, Sheng ${ }^{b}$ \\ Wei, Xianyong*, Zong, Zhimin ${ }^{a}$ \\ $\left({ }^{a}\right.$ School of Chemical Engineering and Technology, China University of Mining and Technology, \\ Xuzhou 221008) \\ ( ${ }^{b}$ College of Chemistry and Chemical Engineering, Xuzhou Normal University Xuzhou 221116)
}

\begin{abstract}
An efficient and convenient method for the preparation of 3-carbonitrile pyridin-2(1H)-one derivative by the one-pot reaction of aromatic aldehydes, acetones and 2-cyanoacetamide in the presence of sodium hydroxide under solvent-free condition was reported. This method has the advantages of excellent yields, mild reaction conditions, easy work-up, and environmentally friendly procedure.
\end{abstract}

Keywords pyridin-2(1H)-one; heterocycle; solvent-free reaction; synthesis

在合成药物的发展过程中, 杂环化合物是药物创制 的主流. 近年来, 世界专利 $90 \%$ 以上是杂环化合物, 其 重要的原因是杂环化合物的药物具有 “高效、低毒、安 全” 的特点. 杂环化合物正是由于应用性极强, 发展极 为迅速, 所以深受人们的青睐. 吡啶是重要杂环化合物, 也是很多天然化合物的核心结构之一, 越来越多的研究 表明吡啶类化合物具有消炎、止痛、退热、抑菌、杀菌、 抗高血糖、抗癌、抗凝血剂等药理活性. 鉴于吡啶化合 物在有机合成以及药物中的重要性, 有机合成化学家对 吡啶的合成与应用进行了广泛的研究 ${ }^{[1 \sim 4]}$. 2-吡啶酮又 是吡啶重要的衍生物, 同样具有广泛的生理及药理活性 而被用于药物化学 ${ }^{[5]}$. 研究发现有些吡啶酮衍生物是治 疗心脏病的强心剂 ${ }^{[6]}$, 有些是蛋白质的抑制剂 ${ }^{[7]}$, 有些 化合物还被用于临床治疗癌症的药物 ${ }^{[8]}$, 还有些化合物
具有抗菌活性. 此类化合物最典型的合成方法是芳醛与 丙酮 $1: 1$ 缩合产物 $(\alpha, \beta$-不饱和酮)与氭乙酰胺反应得 到 ${ }^{[8]}$, 已报道的反应条件主要有 THF 为溶剂, DBU 和 $\mathrm{MnO}_{2}$ 为催化剂; $\mathrm{DMSO}$ 为溶剂, 叔丁醇钾作催化剂; $\mathrm{EtOH}$ 为溶剂, $\mathrm{Et}_{3} \mathrm{~N}$ 或 $\mathrm{C}_{5} \mathrm{H}_{11} \mathrm{~N}$ 作催化剂等. 其他的合成 方法也有报道，比如芐澳、1,2-环氧丙烷、氧乙酰胺和 苯磺酸钠反应来制备吡啶酮 ${ }^{[9]} ; \alpha, \beta$-不饱和酮与取代氰 乙酰胺在异丙醇溶剂中六氢吡啶催化并在微波辐射下 得到产物 ${ }^{[10]},[2+2+2]$ 环加成反应得到产物 ${ }^{[1]}$, 乙酰基 取代的乙酰乙酸乙酯与醛、乙酸铵反应得到产物 ${ }^{[12]}$ 和 $\alpha, \beta$-不饱和酸酯与丙二睛或者㲵乙酸酯反应来制备产 物 ${ }^{[13]}$ 等. 这些方法合成过程中有机溶剂是必不可少的, 而且有些还是多步反应，产率较低，后处理复杂，所以 寻找新的方法来合成吡啶酮衍生物是必要的.

\footnotetext{
*E-mail: 1crong@xznu.edu.cn or 1crong2005@yahoo.com

Received April 14, 2011; revised July 21, 2011; accepted October 25, 2011.

Project supported by the Natural Science Foundation of Jiangsu Education Department (No. 08KJB150017), and the Research Platform Foundation of Xuzhou Normal University (No. 10XLS02).

江苏省高校自然科学基础研究基金(No. 08KJB150017)、徐州师范大学科研平台(No. 10XLS02)资助项目.
} 
无溶剂有机合成 ${ }^{[14 ~ 19]}$ 由于在反应过程中不使用有 机溶剂, 具有反应速度快、反应选择性好、处理简单, 还 可以得到一些常规液相反应得不到的产物而受到人们 广泛关注. 本文报道一种无溶剂条件下合成 3-氭基-2吡啶酮衍生物的方法, 反应见 Eq. 1.

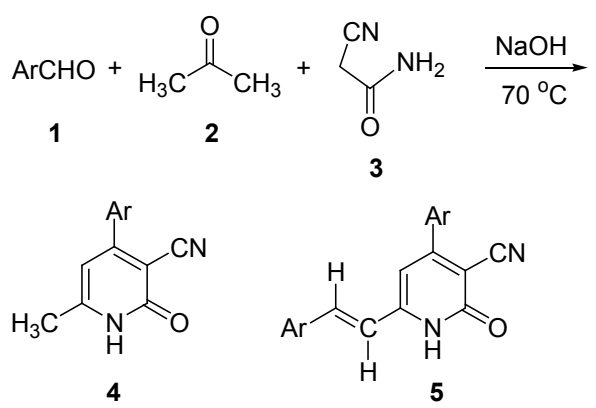

4a: $\mathrm{Ar}=\mathrm{C}_{6} \mathrm{H}_{5}, \mathbf{4 b}: \mathrm{Ar}=4-\mathrm{CH}_{3} \mathrm{C}_{6} \mathrm{H}_{4}, \mathbf{4 c}: \mathrm{Ar}=3,4-\left(\mathrm{CH}_{3} \mathrm{O}\right)_{2} \mathrm{C}_{6} \mathrm{H}_{3}$, 4d: $\mathrm{Ar}=4-\mathrm{BrC}_{6} \mathrm{H}_{4}, 4 \mathrm{e}: \mathrm{Ar}=3,4-\mathrm{Cl}_{2} \mathrm{C}_{6} \mathrm{H}_{3} ; 5 \mathrm{a}: \mathrm{Ar}=4-\mathrm{ClC}_{6} \mathrm{H}_{4}$, 5b: $\mathrm{Ar}=4-\mathrm{FC}_{6} \mathrm{H}_{4}, \mathbf{5 c}: \mathrm{Ar}=3,4-\mathrm{OCH}_{2} \mathrm{OC}_{6} \mathrm{H}_{3}, \mathbf{5 d}: \mathrm{Ar}=4-\mathrm{CH}_{3} \mathrm{OC}_{6} \mathrm{H}_{4}$

\section{1 实验部分}

\section{1 仪器和试剂}

玛瑙研针; 熔点测定使用北京科仪电光仪器厂生产 的 TX5 显微熔点仪测定(温度未经校正); 红外光谱采用 FT/IR-8101 型红外光谱仪测定 $(\mathrm{KBr}$ 压片); 核磁共振氢 谱采用 Bruker-400 MHz 型核磁共振仪测定, DMSO- $d_{6}$ 为溶剂, TMS 为内标; 元素分析用 Perkin-Elmer 2400II 型元素分析仪测定; 高分辨质谱采用 Bruker micrOTOFQ-II 高分辨质谱仪测定; 所用试剂均为分析纯或化学 纯，使用前未经纯化.

\subsection{3 -氰基-2-吡啶酮衍生物 4 和 5 的合成}

芳醛 $(2 \mathrm{mmol})$ 、丙酮 $(3 \mathrm{mmol}) 、$ 㲵乙酰胺 $(2 \mathrm{mmol})$ 和 $\mathrm{NaOH}(0.1 \mathrm{~g})$ 置于玛瑙研钭中混合, 反应物迅速转移 到 $50 \mathrm{~mL}$ 圆底烧瓶中, $70{ }^{\circ} \mathrm{C}$ 下反应 $20 \mathrm{~min}$, 得黄色固 体, 用水洗至中性, 抽滤, 固体粗产物用 $95 \% \mathrm{EtOH}$ 重 结晶，干燥，得目标化合物 $\mathbf{4}$ 或 $\mathbf{5}$.

3-氧基-4-苯基-6-甲基-1,2-二氢吡啶-2-酮(4a)：黄色 固体, 收率 80\%. m.p. $276 \sim 277{ }^{\circ} \mathrm{C} ;{ }^{1} \mathrm{H}$ NMR $(400 \mathrm{MHz}$, DMSO- $\left.d_{6}\right) \delta: 2.32\left(\mathrm{~s}, 3 \mathrm{H}, \mathrm{CH}_{3}\right), 6.58\left(\mathrm{~s}, 1 \mathrm{H}, \mathrm{C}^{5}-\mathrm{H}\right), 7.56$ (s, 3H, ArH), 7.60 (s, 2H, ArH), $12.70(\mathrm{~s}, 1 \mathrm{H}, \mathrm{NH})$; IR (KBr) v: 3290, 3016, 2900, 2854, 2804, 2682, 2221, 1660, $1625,1475,1386,1228,939,853,756,693 \mathrm{~cm}^{-1}$. Anal. calcd for $\mathrm{C}_{13} \mathrm{H}_{10} \mathrm{~N}_{2} \mathrm{O}$ : C 74.27, $\mathrm{H} \mathrm{4.79,} \mathrm{N} 13.33$; found $\mathrm{C}$ 74.40, H 4.84, N 13.41. HRMS calcd for $\mathrm{C}_{13} \mathrm{H}_{10} \mathrm{~N}_{2} \mathrm{O}[\mathrm{M}+$ $\mathrm{H}]^{+}: 211.0871$, found 211.0871 .

3-氧基-4-对甲基苯基-6-甲基-1,2-二氢吡啶-2-酮 (4b): 黄色固体, 收率 70\%. m.p. $267 \sim 268{ }^{\circ} \mathrm{C} ;{ }^{1} \mathrm{H}$ NMR
(400 MHz, DMSO- $\left.d_{6}\right) \delta: 2.31\left(\mathrm{~s}, 3 \mathrm{H}, \mathrm{CH}_{3}\right), 2.39$ (s, 3H, $\left.\mathrm{CH}_{3}\right), 6.33\left(\mathrm{~s}, 1 \mathrm{H}, \mathrm{C}^{5}-\mathrm{H}\right), 7.35(\mathrm{~d}, J=8.0 \mathrm{~Hz}, 2 \mathrm{H}, \mathrm{ArH})$, 7.51 (d, $J=8.0 \mathrm{~Hz}, 2 \mathrm{H}, \mathrm{ArH}), 12.57$ (s, 1H, NH); IR (KBr) $v: 3293,3008,2844,2782,2673,2216,1649,1613,1475$, 1339, 1214, 932, 825, $651 \mathrm{~cm}^{-1}$. Anal. calcd for $\mathrm{C}_{14} \mathrm{H}_{12} \mathrm{~N}_{2} \mathrm{O}$ : C 74.98, H 5.39, N 12.49; found C 74.55, H 5.44, N 12.43. HRMS calcd for $\mathrm{C}_{14} \mathrm{H}_{12} \mathrm{~N}_{2} \mathrm{O}[\mathrm{M}+\mathrm{H}]^{+}$: 225.1028 , found 225.1038 .

3-氰基-4-(3,4-二甲氧基苯基)-6-甲基-1,2-二氢吡啶2-酩(4c): 黄色固体, 收率 76\%. m.p. $>280{ }^{\circ} \mathrm{C} ;{ }^{1} \mathrm{H}$ NMR (400 MHz, DMSO- $\left.d_{6}\right) \delta: 2.31\left(\mathrm{~s}, 3 \mathrm{H}, \mathrm{CH}_{3}\right), 3.83(\mathrm{~s}, 3 \mathrm{H}$, $\left.\mathrm{OCH}_{3}\right), 3.84\left(\mathrm{~s}, 3 \mathrm{H}, \mathrm{OCH}_{3}\right), 6.39\left(\mathrm{~s}, 1 \mathrm{H}, \mathrm{C}^{5}-\mathrm{H}\right), 7.11(\mathrm{~d}$, $J=8.0 \mathrm{~Hz}, 1 \mathrm{H}, \mathrm{ArH}), 7.23$ (d, $J=8.0 \mathrm{~Hz}, 2 \mathrm{H}, \mathrm{ArH}), 12.48$ (s, 1H, NH); IR (KBr) v: 3303, 3002, 2935, 2836, 2214, $1665,1631,1522,1456,1327,1286,1145,935,802,780$ $672,621 \mathrm{~cm}^{-1}$. Anal. calcd for $\mathrm{C}_{15} \mathrm{H}_{14} \mathrm{~N}_{2} \mathrm{O}_{3}: \mathrm{C} 66.66, \mathrm{H}$ 5.22, N 10.36; found $\mathrm{C} 66.75, \mathrm{H} 5.26, \mathrm{~N}$ 10.29. HRMS calcd for $\mathrm{C}_{15} \mathrm{H}_{14} \mathrm{~N}_{2} \mathrm{O}_{3}[\mathrm{M}+\mathrm{H}]^{+}$: 271.1083, found 271.1080 .

3-氧基-4-对溴苯基-6-甲基-1,2-二氢吡啶-2-酮(4d): 黄色固体, 收率 $80 \%$. m.p. $250 \sim 252{ }^{\circ} \mathrm{C} ;{ }^{1} \mathrm{H}$ NMR $(400$ MHz, DMSO- $\left.d_{6}\right) \delta: 2.31\left(\mathrm{~s}, 3 \mathrm{H}, \mathrm{CH}_{3}\right), 6.34\left(\mathrm{~s}, 1 \mathrm{H}, \mathrm{C}^{5}-\mathrm{H}\right)$, 7.56 (dt, $J=2.0,4.4 \mathrm{~Hz}, 2 \mathrm{H}, \mathrm{ArH}), 7.76$ (dt, $J=2.0,2.4$ $\mathrm{Hz}, 2 \mathrm{H}, \mathrm{ArH}), 12.67$ (s, 1H, NH); IR (KBr) v: 3293, 3012, 2896, 2851, 2798, 2679, 2219, 1659, 1624, 1473, 1386, 1341, 1012, 935, 821, 779, $586 \mathrm{~cm}^{-1}$. Anal. calcd for $\mathrm{C}_{13} \mathrm{H}_{9} \mathrm{BrN}_{2} \mathrm{O}$ : C 54.00, H 3.14, N 9.69; found C 54.17, H 3.17, N 9.60. HRMS calcd for $\mathrm{C}_{13} \mathrm{H}_{9} \mathrm{BrN}_{2} \mathrm{O}[\mathrm{M}+\mathrm{H}]^{+}$: 288.9977, found 288.9976 .

3-氧基-4-(3,4-二氯苯基)-6-甲基-1,2-二氢吡啶-2-酮 (4e): 黄色固体, 收率 78\%. m.p. $>280{ }^{\circ} \mathrm{C} ;{ }^{1} \mathrm{H}$ NMR (400 MHz, DMSO- $\left.d_{6}\right) \delta: 2.32\left(\mathrm{~s}, 3 \mathrm{H}, \mathrm{CH}_{3}\right), 6.40\left(\mathrm{~s}, 1 \mathrm{H}, \mathrm{C}^{5}-\mathrm{H}\right)$, 7.61 (dd, $J=2.0,2.0 \mathrm{~Hz}, 1 \mathrm{H}, \mathrm{ArH}), 7.83$ (d, $J=8.4 \mathrm{~Hz}$, $1 \mathrm{H}, \mathrm{ArH}), 7.89$ (d, $J=2.0 \mathrm{~Hz}, 1 \mathrm{H}, \mathrm{ArH}), 12.73$ (s, $1 \mathrm{H}$, $\mathrm{NH})$; IR (KBr) v: 3289, 3011, 2896, 2850, 2797, 2222, 1656, 1624, 1473, 1385, 1340, 1227, 1148, 1034, 850, 825, $658,593 \mathrm{~cm}^{-1}$. Anal. calcd for $\mathrm{C}_{13} \mathrm{H}_{8} \mathrm{Cl}_{2} \mathrm{~N}_{2} \mathrm{O}$ : C 55.94, $\mathrm{H}$ 2.89, N 10.04; found C 55.75, H 2.87, N 10.09. HRMS calcd for $\mathrm{C}_{13} \mathrm{H}_{8} \mathrm{Cl}_{2} \mathrm{~N}_{2} \mathrm{O}[\mathrm{M}+\mathrm{H}]^{+}:$279.0092, found 279.0092 .

3-氧基-4-对氯苯基-6-对氯苯乙烯基-1,2-二氢吡啶2-酮 (5a): 淡黄色固体, 收率 $81 \%$. m.p. $>290{ }^{\circ} \mathrm{C} ;{ }^{1} \mathrm{H}$ NMR (400 MHz, DMSO- $\left.d_{6}\right) \delta: 6.83\left(\mathrm{~s}, 1 \mathrm{H}, \mathrm{C}^{5}-\mathrm{H}\right), 7.10(\mathrm{~d}$, $J=16.8 \mathrm{~Hz}, 1 \mathrm{H}, \mathrm{CH}=\mathrm{CH}), 7.53(\mathrm{~d}, J=8.8 \mathrm{~Hz}, 2 \mathrm{H}, \mathrm{ArH})$, $7.60(\mathrm{~d}, J=8.4 \mathrm{~Hz}, 2 \mathrm{H}, \operatorname{ArH}), 7.66(\mathrm{~d}, J=8.8 \mathrm{~Hz}, 2 \mathrm{H}$, 
ArH), 7.71 (d, $J=8.4 \mathrm{~Hz}, 2 \mathrm{H}, \operatorname{ArH}), 7.84$ (d, $J=16.8 \mathrm{~Hz}$, $1 \mathrm{H}, \mathrm{CH}=\mathrm{CH}), 12.65$ (s, 1H, NH); IR (KBr) v: 3131, 3093, 3012, 2954, 2900, 2851, 2798, 2221, 1661, 1626, 1597, 1527, 1497, 1475, 1387, 1341, 1225, 1125, 1096, 1048, 1016, 939, 826, 780, 650, $590 \mathrm{~cm}^{-1}$. Anal. calcd for $\mathrm{C}_{20} \mathrm{H}_{12} \mathrm{Cl}_{2} \mathrm{~N}_{2} \mathrm{O}$ : C 65.41, H 3.29, N 7.63; found C 65.56, H 3.24, $\mathrm{N}$ 7.57. HRMS calcd for $\mathrm{C}_{20} \mathrm{H}_{12} \mathrm{Cl}_{2} \mathrm{~N}_{2} \mathrm{O}[\mathrm{M}+\mathrm{H}]^{+}$: 367.0405 , found 367.0401 .

3-氧基-4-对氟苯基-6-对氟苯乙烯基-1,2-二氢吡啶2-酮 (5b): 淡黄色固体, 收率 $85 \%$. m.p. $>280{ }^{\circ} \mathrm{C} ;{ }^{1} \mathrm{H}$ NMR (400 MHz, DMSO- $\left.d_{6}\right) \delta: 6.82\left(\mathrm{~s}, 1 \mathrm{H}, \mathrm{C}^{5}-\mathrm{H}\right), 7.04$ (d, $J=16.8 \mathrm{~Hz}, 1 \mathrm{H}, \mathrm{CH}=\mathrm{CH}), 7.32(\mathrm{t}, J=8.4 \mathrm{~Hz}, 2 \mathrm{H}, \mathrm{ArH})$, $7.44(\mathrm{t}, J=8.4 \mathrm{~Hz}, 2 \mathrm{H}, \operatorname{ArH}), 7.65$ (q, $J=7.6 \mathrm{~Hz}, 2 \mathrm{H}$, $\operatorname{ArH}), 7.76$ (q, $J=7.6 \mathrm{~Hz}, 2 \mathrm{H}, \operatorname{ArH}), 7.85$ (d, $J=16.8 \mathrm{~Hz}$, $1 \mathrm{H}, \mathrm{CH}=\mathrm{CH}), 12.62(\mathrm{~s}, 1 \mathrm{H}, \mathrm{NH})$; IR $(\mathrm{KBr}) v: 3135,3017$, 2904, 2854, 2803, 2221, 1660, 1626, 1609, 1519, 1475, 1387, 1343, 1316, 1249, 1231, 1169, 1049, 834, 803, 781, $678,651,606 \mathrm{~cm}^{-1}$. Anal. calcd for $\mathrm{C}_{20} \mathrm{H}_{12} \mathrm{~F}_{2} \mathrm{~N}_{2} \mathrm{O}: \mathrm{C} 71.85$, $\mathrm{H}$ 3.62, N 8.38; found C 71.66, H 3.68, N 8.29. HRMS calcd for $\mathrm{C}_{20} \mathrm{H}_{12} \mathrm{~F}_{2} \mathrm{~N}_{2} \mathrm{O}[\mathrm{M}+\mathrm{H}]^{+}$: 335.0996, found 335.0998 .

3-氧基-4-(5-苯并 $[d][1,3]$-二氧杂环戊烯基)-6-(5-苯 并 $[d][1,3]$-二氧杂环戊烯基乙烯基)-1,2-二氢吡啶-2-酮 (5c): 淡黄色固体, 收率 $75 \%$. m.p. $>290{ }^{\circ} \mathrm{C} ;{ }^{1} \mathrm{H}$ NMR (400 MHz, DMSO-d $\left.d_{6}\right) \delta: 6.10\left(\mathrm{~s}, 2 \mathrm{H}, \mathrm{OCH}_{2} \mathrm{O}\right.$ ), 6.15 (s, $\left.2 \mathrm{H}, \mathrm{OCH}_{2} \mathrm{O}\right), 6.71\left(\mathrm{~s}, 1 \mathrm{H}, \mathrm{C}^{5}-\mathrm{H}\right), 6.91(\mathrm{~d}, J=16.4 \mathrm{~Hz}, 1 \mathrm{H}$, $\mathrm{CH}=\mathrm{CH}), 6.98(\mathrm{~d}, J=8.4 \mathrm{~Hz}, 1 \mathrm{H}, \mathrm{ArH}), 7.07$ (d, $J=8.0$ $\mathrm{Hz}, 1 \mathrm{H}, \mathrm{ArH}), 7.11$ (d, $J=8.0 \mathrm{~Hz}, 1 \mathrm{H}, \mathrm{ArH}), 7.21(\mathrm{~s}, 1 \mathrm{H}$, ArH), 7.27 (s, 1H, ArH), 7.68 (d, $J=8.4 \mathrm{~Hz}, 1 \mathrm{H}, \operatorname{ArH})$, $7.77(\mathrm{~d}, J=16.4 \mathrm{~Hz}, 1 \mathrm{H}, \mathrm{CH}=\mathrm{CH}), 12.45(\mathrm{~s}, 1 \mathrm{H}, \mathrm{NH})$; IR (KBr) $v$ : 3135, 3095, 2916, 2219, 1653, 1622, 1599, 1542, 1526, 1506, 1477, 1456, 1333, 1270, 1259, 1248, 1197, 1094, 1045, 957, 929, 885, 843, 805, 786, $670 \mathrm{~cm}^{-1}$. Anal. calcd for $\mathrm{C}_{22} \mathrm{H}_{14} \mathrm{~N}_{2} \mathrm{O}_{5}$ : C 68.39, H 3.65, N 7.25; found $\mathrm{C}$ 68.50, H 3.60, N 7.33. HRMS calcd for $\mathrm{C}_{22} \mathrm{H}_{14} \mathrm{~N}_{2} \mathrm{O}_{5}[\mathrm{M}+$ $\mathrm{H}]^{+}$: 387.0981, found 387.0980.

3-氧基-4-对甲氧基苯基-6-对甲氧基苯乙烯基-1,2二氢吡啶-2-酮 (5d): 淡黄色固体, 78\%. m.p. $>290{ }^{\circ} \mathrm{C} ;{ }^{1} \mathrm{H}$ NMR (400 MHz, DMSO- $d_{6}$ ) $\delta: 3.77\left(\mathrm{~s}, 3 \mathrm{H}, \mathrm{OCH}_{3}\right), 3.80$ (s, 3H, $\left.\mathrm{OCH}_{3}\right), 5.79(\mathrm{~d}, J=16.4 \mathrm{~Hz}, 1 \mathrm{H}, \mathrm{CH}=\mathrm{CH}), 6.92$ (s, 1H, C $-\mathrm{H}), 6.93$ (d, $J=8.8 \mathrm{~Hz}, 2 \mathrm{H}, \mathrm{ArH}), 7.01$ (d, $J=$ $8.8 \mathrm{~Hz}, 2 \mathrm{H}, \mathrm{ArH}), 7.21$ (d, $J=8.8 \mathrm{~Hz}, 2 \mathrm{H}, \mathrm{ArH}), 7.40$ (d, $J=8.8 \mathrm{~Hz}, 2 \mathrm{H}, \mathrm{ArH}), 8.1(\mathrm{~d}, J=16.4 \mathrm{~Hz}, 1 \mathrm{H}, \mathrm{CH}=\mathrm{CH})$, 12.52 (s, 1H, NH); IR (KBr) v: 3150, 3019, 2934, 2838, 2219, 1650, 1627, 1605, 1587, 1511, 1475, 1460, 1426,
1363, 1301, 1253, 1204, 1179, 1113, 1030, 967, 896, 822, $667,643 \mathrm{~cm}^{-1}$. Anal. calcd for $\mathrm{C}_{22} \mathrm{H}_{18} \mathrm{~N}_{2} \mathrm{O}_{3}: \mathrm{C} 73.73, \mathrm{H}$ 5.06, N 7.82; found C 73.85, H 5.11, N 7.76. HRMS calcd for $\mathrm{C}_{22} \mathrm{H}_{18} \mathrm{~N}_{2} \mathrm{O}_{3}[\mathrm{M}+\mathrm{H}]^{+}: 359.1396$, found 359.1403.

\section{2 结果和讨论}

无溶剂反应由于其温和的反应条件，良好的产量, 简单的处理方式而受到人们的广泛的关注, 采用此方法 合成化合物的文献近年来呈快速增长的趋势, 每年有大 量的重要的论文被报道. 本文就是采用无溶剂的方法合 成系列 3-氧基-2-吡啶酮衍生物. 只需将醛、丙酮和氧乙 酰胺在研钵中混合均匀, 加入 $0.1 \mathrm{~g} \mathrm{NaOH}$, 继续研磨成 固体, 转移到圆底烧瓶中加热, 于 $70{ }^{\circ} \mathrm{C}$ 反应 $20 \mathrm{~min}$ 左 右即可完成反应, 得到黄色或淡黄色固体, 用水洗至中 性，再用 $95 \%$ 乙醇重结晶即可得到目标产物 4 或 $\mathbf{5}$. 在 反应中我们发现一个奇怪的现象, 用不同的醛反应时得 到的产物结构并不一样, 因为在产物的核磁共振谱中有 的化合物在 $\delta 2.31$ 附近有一个 3 个氢的单峰, 这明显是 甲基 $\left(\mathrm{CH}_{3}\right)$ 的峰, 而另外一些化合物中并没有发现这个 峰, 但却在 $\delta 6.90$ 和 7.70 处出现两个双峰, 且耦合常数 较大 $(J=16.4 \mathrm{~Hz})$, 根据核磁的知识, 这应是双键碳上氢 原子的信号，且是反式结构，考虑到结构中没有甲基的 信号, 我们认为是结构 $\mathbf{4}$ 中的甲基与另外一个分子醛反 应得到烯烃结构, 这个推测在产物 $\mathbf{5 d}$ 上得到了验证, 我们在其核磁谱明显发现到两个甲氧基的峰，分别在 $\delta$ 3.77 和 3.80 处有两个 3 个氢的单峰，同时在化合物 $\mathbf{5 c}$ 上我们也见到了两个亚甲基的单峰 $\left(\mathrm{OCH}_{2} \mathrm{O}\right)$, 分别在 $\delta$ 6.10 和 6.15 处. 说明分子中确实是两分子醛出现在结构 中. 为了进一步对产物的结构进行确证，我们对所有合 成的化合物做了高分辨质谱分析, 所有化合物得到的数 据与计算值是十分吻合的, 这说明 $\mathbf{4}$ 和 $\mathbf{5}$ 的结构是正确 的. 我们还想考察化合物 5 在液相的是否能够合成, 遗 憾的是我们尝试不同溶剂如甲醇、乙醇、DMF 等和不 同催化剂如 $\mathrm{KOH}, \mathrm{NaOH}, \mathrm{EtONa}$ 和 $t$-BuOK, 但都没有 得到 5 的结构，而化合物 $\mathbf{4}$ 却可以得到，但产率较低，这 说明无溶剂条件是合成化合物 $\mathbf{4}$ 和 $\mathbf{5}$ 的有效的方法，特 别是对化合物 5 来说更是如此. 当然对反应中为什么不 同醛得到的产物结构不同, 我们还在研究, 目前还没有 找到令人信服的理由. 产物的结构在红外光谱中也有明 显的体现, 比如氨基( $\mathrm{NH}$ )的特征伸缩振动吸收峰 $\mathbf{4}$ 出现 在 $3330 \mathrm{~cm}^{-1}$ 附近, 而 5 出现在 $3130 \mathrm{~cm}^{-1}$ 附近, 氭基尖 锋出现在 $2220 \mathrm{~cm}^{-1}$ 附近, 羰基 $(\mathrm{C}=\mathrm{O})$ 强的伸缩振动吸 收峰出现在 $1660 \mathrm{~cm}^{-1}$ 附近. 在氢谱中, 氨基质子的吸 收峰在 $\delta 12.50$ 附近出现, 新生成的吡啶环上 $\mathrm{C}^{5}-\mathrm{H}$ 单峰 在 $\delta 6.50 \sim 6.90$ 之间出现. 芳氢原子的个数与结构也是 
吻合的. 由此可见无溶剂条件是合成此类化合物的一条 简单、方便和环境友好的合成方法.

\section{References}

[1] Cottineau, B.; Toto, P.; Marot, C.; Pipaud, A.; Chenault, J. Bioorg. Med. Chem. Lett. 2002, 12, 2105.

[2] Lee, K. Y.; Kim, J. M.; Kim, J. N. Tetrahedron Lett. 2003, 44, 6737.

[3] Jia, Z. J.; Wu, Y.; Huang, W.; Zhang, P.; Song, Y.; Woolfrey, J.; Sinha, U.; Arfsten, A.; Edwards, S.; Hutchaleelaha, A.; Hollennbach, S.; Lambing, J.; Scarborough, R.; Zhu, B. Y. Bioorg. Med. Chem. Lett. 2004, 14, 1229.

[4] John, W. L.; Patera, R. M.; Plunner, M. J.; Halling, B. P.; Yuhas, D. A. Pestic. Sci. 1994, 42, 29.

[5] (a) O'Hagan, D. Nat. Prod. Rep. 2000, 17, 435.

(b) Bailey, P. D.; Millwood, P. A.; Smith, P. D. Chem. Commun. 1998, 633.

[6] Pastelin, G.; Mendez, R.; Kabela, E; Farah, A. Life Sci. 1983, 33, 1787.

[7] Angibaud, P. R.; Venet, M. G.; Filliers, W.; Broeckx, R.; Ligny, Y. A.; Muller, P.; Poncelet, V. S.; End, D. W. Eur. J. Org. Chem. 2004, 479 .

[8] (a) Huth, A.; Schmiechen, R.; Moto, L.; Beetz, I.; Breitkopf, A.;
Frost, E.; Schumann, I.; Thielert, K. Arch. Pharm. 1988, 321, 297. (b) Carles, L.; Narkunan, K.; Penlou, S.; Rousset, L.; Bouchu, D.; Ciufolini, M. A. J. Org. Chem. 2002, 67, 4304.

[9] Li, W. W.; Chen, Y.; Lam, Y. Tetrahedron Lett. 2004, 45, 6545.

[10] Gorobert, N. Y.; Yousefi, B. H.; Belaj, F.; Kaffe, C. O. Tetrahedron 2004, 60, 8633.

[11] Oberg, K. M.; Lee, E. E.; Rovis, T. Tetrahedron 2009, 69, 5056.

[12] Svetlik, J.; Kettmann, V.; Zaleska, B. Tetrahedron Lett. 2005, 46, 5511.

[13] Mont, N.; Teixido, J.; Borrell, J. I.; Kappe, C. O. Tetrahedron Lett. 2003, 44, 5385.

[14] Laschat, S.; Dickner, T. Synthesis 2000, 1781.

[15] Tanaka, T.; Toda, F. Chem. Rev. 2000, 100, 1025.

[16] Rong, L. C.; Han, H. X.; Jiang. H.; Tu, S. J. Synth. Commun. 2009, 39, 1027.

[17] Gao, L. J.; Ji, H. L.; Rong, L. C.; Han, H. X.; Shi, Y. H.; Tu, S. J. J. Heterocycl. Chem., 2010, 47, 358.

[18] Zhang, G. P.; Xia, Y. Chin. J. Org. Chem. 2010, 30, 449 (in Chinese). (张国平, 夏燕, 有机化学, 2010, 30, 449.)

[19] Yu Y.; Guo, H. Y. Chin. J. Org. Chem. 2011, 31, 96 (in Chinese). (余意, 郭红云, 有机化学, 2011, 31, 96.)

(Sun, H.; Fan, Y.) 